\title{
Design And Implementation Of The Cloud Intelligence Office System Based On IOT
}

\author{
Chen Hongyan*, Wan Junwei*, Zhao Jing ${ }^{*}$ \\ *.Beijing Institute of Tracking and Telecommunicaitons Technology, Beijing 100094, China \\ ${ }^{+}$. Nanjing University of Science and Technology School of Computer Science and Engineering, Nanjing 210094, China \\ child_smile@163.com
}

Keywords: IOT; Cloud computing; Office system.

\begin{abstract}
Currently, there are many independent and closed "chimney" application systems in enterprises, In view of this situation, this paper designs and implements an private cloud intelligence office system based on IOT, which focused on the Integration of all servers, storage, network resources, and take cloud computing technology, big data storage technology, network connection technology, database technology, networking technology as core. The methods and techniques realized the unified management of the office integrated information system, with strong safety, practicality, accessibility, expansibility. The results show that the design improved the collaboration and resource sharing, and created a more scientific office environment and humane working conditions.
\end{abstract}

\section{Introduction}

In recent years,IOT(Internet of things), big data and artificial intelligence are widely used, which have brought great and far-reaching influence to the development of information industry, infrastructure is innovated, and cloud computing has developed to a new stage.With the intelligent building, smart home become a hot topic in the industry, the intelligent office system relying on IOT, cloud computing and other information technology is quietly come into our sight, and it integrated information collection, information maintenance, business processes, materials management, security and the essence of portals.

This paper presents the cloud intelligence office integrated information management system based on IOT, and through the self-controlled domestic architecture, the system built the private cloud platform to integrate all servers, storage and cyber source. Use of cloud computing technology, big data storage technology, network connection technology, database technology and IOT (include sensor technology, RFID(Radio Frequency Identification) technology, Zigbee technology, monitoring technology,communication technology, global positioning technology and so on), the system achieved office dynamics of integrated information management, networking of business processes, automation of physical environment and safety and intelligent of materials management and maintenance, so as to enhance the management efficiency and work quality, improve the office conditions, optimize business processes, reduce operating costs, and improve work efficiency.

\section{Present situation analysis}

Office management system is the core of the information integration management of the academy of science and University Research Institute. With the development of information technology, such as equipment management, asset management, access control, video surveillance, environmental monitoring and other business subsystem has independent.

A University Research Institute, Institute of information management, with the development of information technology, such as equipment management, asset management, access control, video surveillance, environmental monitoring and other business system has independent construction and accumulation, self-contained application systems and their respective servers and storage devices is independent, servers is sprawl, and the application is scatter, which generated "chimneys"data inlands of information. The chimney type application system leads to the following problems:

1) Business System more landing,low efficiency;

2) Data information can't be shared.The system are isolated island can't share information,resulting in data redundancy, inconsistency;

3) Slow business response. Scalability is poor,the new application deployment cycle is long can't be quickly and flexible to provide services;

4) Equipment utilization rate is low. the hardware scale is big, the purchasing cost is high, the construction cost is high, the resource utilization rate is low;

5) Availability needs to be improved. The system is not redundant backup,business continuity can't be guaranteed;

6) Management is complex. Large amounts of data and type, decentralized infrastructure hardware, multiple management portals are caused great difficulties to the management and maintenance.

With the passage of time, changing business needs, increasing the business scale and improving the business continuity requirements, various information resources of office system including hardware, software and data was explosive growth, and it resulted in straight up of the management costs and system complexity. Such this kind of highly customized equipment structure is very difficult to combine with the new applications, therefore limits the flexible adjustment ability 
which is necessary for enterprises to adapt to the development of business needs, and limits the ability for enterprises to provide services of responding to emerging market rapidly. The "chimney" application system architecture and isolated data are severely limited the development of enterprises, which has become the business development problem must be solved.

\section{System architecture design}

According to the information isolated island which is formed by the dispersed operation system of the current data center, IOT can effectively solve the problem of resource sharing, data exchanging between each application system and so on.
And through IOT the more important thing is to be able to achieve a single application portal, to enhance the utilization of resources, flexible resource scheduling, on-demand allocation, rapid deployment, etc.

\subsection{Cloud platform architecture design}

Data center server storage is unified managed, and designed with the way of Figure 1 using the basic architecture of the cloud. The entire cloud platform includes infrastructure layer, virtualization layer, cloud service management layer, interface component layer and cloud service presentation layer.

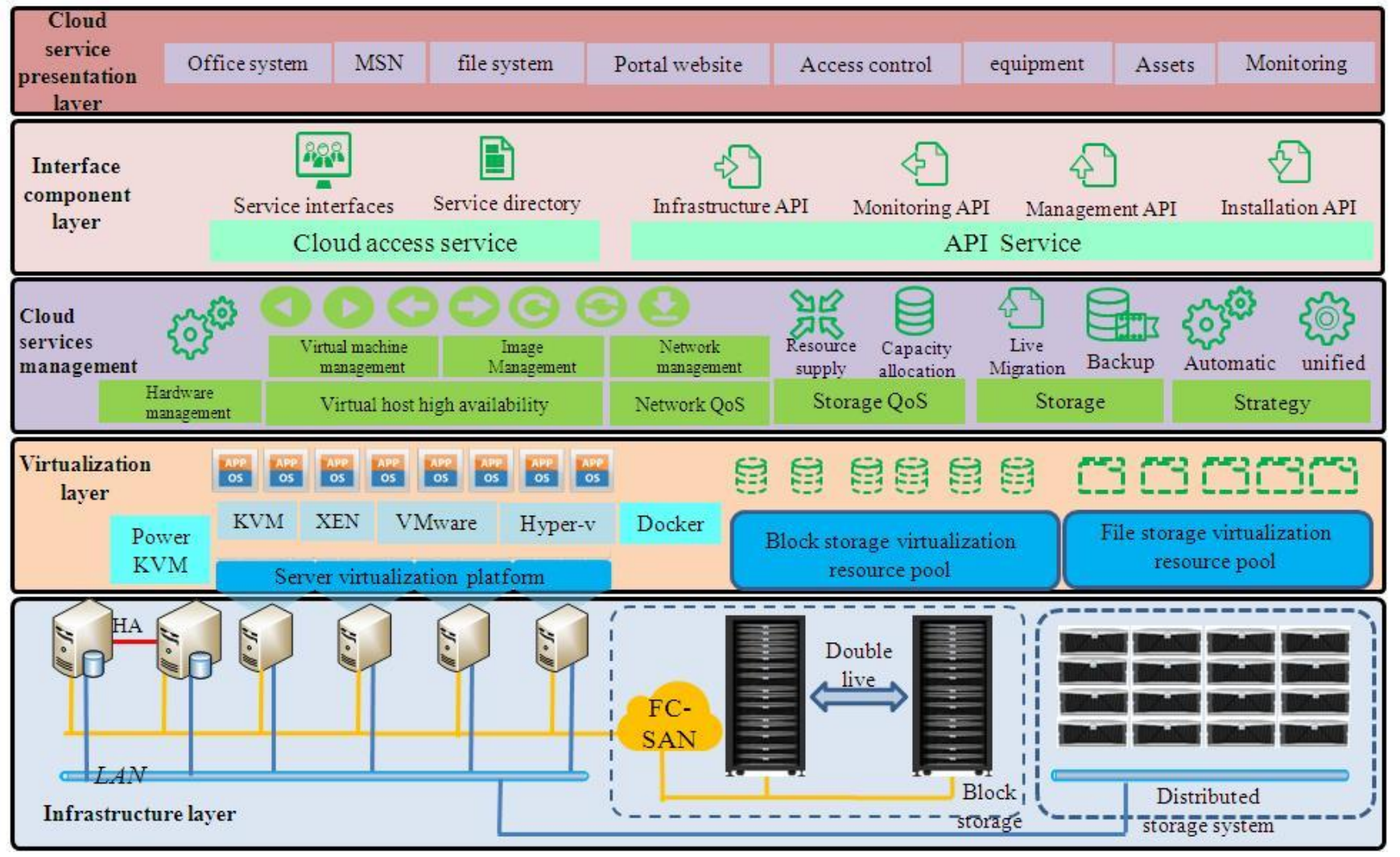

Figure 1:Cloud platform architecture design

As the data center cloud is the basis of IOT,so the existing business system is an essential step in cloud operations. Many of the current cloud data center is based on the Openstack(open cloud computing platform), but the source cloud operating system designed in this paper is simple and crude with Openstack, but is the two development based on Openstack, and adds many unique features.

\subsubsection{Infrastructure layer}

1) Integrated with Openstack

Using the multi controller virtual disk array storage, it is integrated with the Openstack through the Cinder Openstack interface, which can archive the management of volume, and allow process of the volume, volume type and volume snapshot. Cinder does not implement on block equipment management and the actual service,it provides a unified interface for the back-end different storage structure, and different block of equipment suppliers is achieved its driver support in Cinder and integrated with OpenStack.

2) Storage double living mechanism

For the double live storage, multi-controller virtual disk array deployment in a back-to-back way, which realize the integration of all the business system data, and the deployment of the corresponding application in the above. By using the Hyperswap function of the virtual disk array and the volume mirror function, the data can be mirrored in real time, so as to achieve the goal of "dual live, zero switching". So that from the perspective of the whole system architecture, the different server clusters of front-end, SAN (Storage Area Network) network and storage devices, are redundant. What's more important is data real-time image redundancy. At the same time, we can use the upper level technical features, such as virtual machine migration, to achieve dual live of the whole system and even the application. 


\section{3) Data protection}

Although dual live storage has been proposed, but human error, logical failure, etc., still cannot be avoided. So using both NetStor Backup software and virtual tape library to back up is quite necessary. Install the NetStor Backup server software on a virtual machine, install the NetStor Backup client software and the corresponding database proxy software on the virtual server which need backup and backup the data to the virtual tape library. At the same time install SAN proxy on all clients which need backup, to achieve LAN-Free backup. So as to eliminate the backup data in the process of a large number of front-end network occupation.

\subsubsection{Virtualization layer}

To achieve cloud, virtualization is the foundation. Virtualization layer is actually the construction of the resources pool, by using server virtualization technology to integrate server resources of infrastructure layer.

1) Server virtualization

As shown in Figure 2, to achieve server virtualization by KVM (Kernel Virtual Machine). In the pool of server resources, a physical server is divided into multiple virtual servers through virtualization technology, resources are allocated flexible according to the actual needs of the various business systems. It can effectively improve the utilization rate of whole resources. When the business system expires, all virtual machines can be recovered, to avoid the interception and binding of resources.

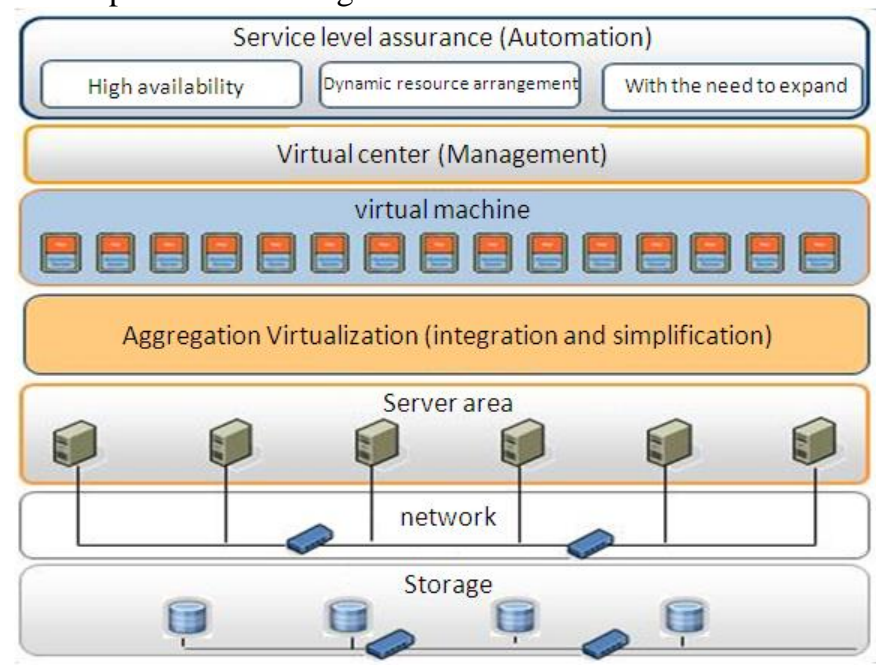

Figure 2:Schematic diagram of virtual server KVM

2) Storage virtualization

Using large capacity cache and advanced caching algorithm of multi-controller virtual disk array, it integrates heterogeneous storage devices from different vendors, shields differences in every storage subsystem supporting, without destroying the existing disk arrays builds the SAN storage pool, and creates virtual disk and supplies storage resource based on storage resource pool. It eliminates limitations and restrictions of the physical storage device architecture, and using of all advanced function of data services, such as lean supply, dynamic layering, snapshots, disaster recovery and double live features, it achieves centralized management and unified resource scheduling of the entire data center storage system.

When the application of file types such as shared files and large data analysis, etc. appears, it can be added in the subsequent storage virtualization resource pool.

\subsubsection{Cloud services management layer}

Cloud services management and virtualization layer is the core of IaaS (Infrastructure as a Service), which is able to provide server storage resources to the appropriate business system. In the cloud service management layer it is mainly divided into three parts, which are the virtual machine service management, network management and storage management services, to provide all the virtual machines or server related services, such as unified entrance, unified management, image management, high availability and disaster recovery, resource supply, capacity allocation, heat transfer, and backup and automatic deployment.

\subsubsection{Interface component layer}

The interface layer is the interface of cloud data center, is the corresponding business system deployment or migration to a virtualized environment, so as to realize the integration of server and storage, enhance the flexibility of the framework, flexibility and accelerate business deployment. And through a variety of interfaces to achieve a variety of resources, such as computing resources, storage resources, as well as the allocation of network resources, the use and deployment of a variety of advanced services such as the realization of.

An essential part of interface layer is the monitoring of cloud data center, such as the monitoring to the performance of physical machine and virtual machine. At the same time, the interface component layer also provides API (Application Programming Interface) to the third party monitoring management vendors for customizing the development and implementation of centralized monitoring and management.

\subsection{Architecture design of IOT}

Architecture design of IOT, is considered to base on the integration and cloud transform of existing chimney type service system data center. So it requires a unified entrance and unified management of various service systems, using hierarchical design of data acquisition layer, data processing layer and application layer, shown as the Schematic diagram of Figure 2. 


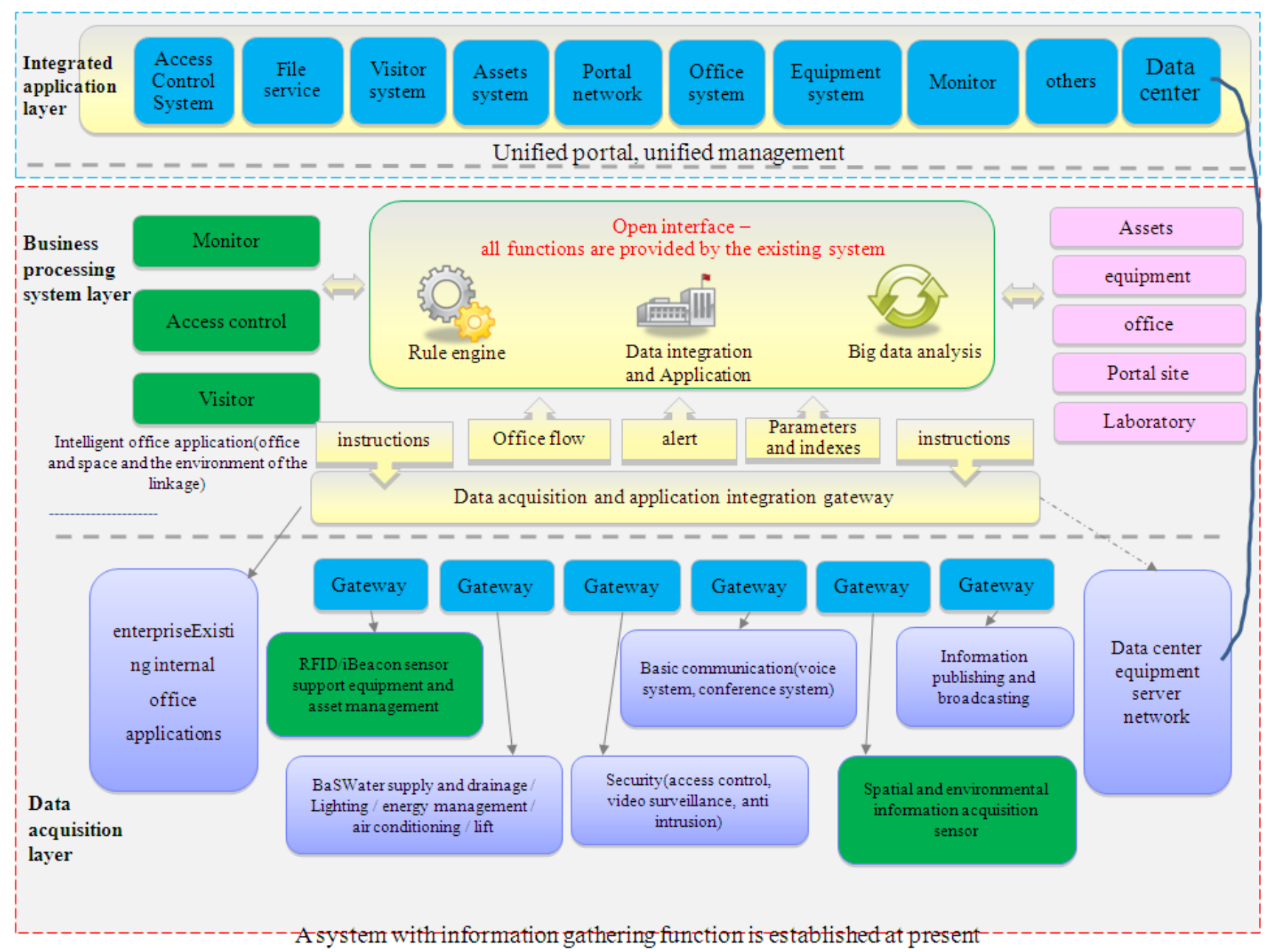

Figure 3: Structure design of IOT

1) Data acquisition layer, using data acquisition of existing system

With various data acquisition equipment, to collect data through technologies of automatic identification (barcode identification, radio frequency identification, image recognition and optical character recognition), wireless transmission (WLAN, WiFi, ZigBee, Bluetooth and Ultrabroadband), global positioning, then transfer and upload data to the cloud data center through the network communication system quickly reliably and safely.

In addition, the data acquisition layer also includes gateway, the communication network and sensing network, and the protocol conversion between different types of networks.

2) Data processing layer, cloud data center

Data processing layer works as the control and decisionmaking layer of IOT. Data acquisition layer collect information in a wide range, and summarize to data processing layer to conduct unified analysis and decision making, so as to achieve real-time monitoring, trend analysis, early warning and linkage etc. Data processing layer includes application support platform sub-layer and application service sub-layer. The application support platform layer provides features of system information sharing, collaboration and interoperability between different applications and systems, including public middleware, information open platform, cloud computing platform and service support platform. Application service sub-layer includes specific business systems, such as access control, resource management, video surveillance, etc.

3) Integrated application layer, unified management of the unified portal of IOT

The different types and levels of chimney type data processing system need different login or authentication methods. Through the integrated and unified portal of IOT to achieve the management and control of all business systems.

\section{Key technology}

1) Unified portal

After the completion of IOT office management platform, all the application systems share the same identity authentication system, to achieve unified portal login, which improve work efficiency greatly. The technical architecture to implement the unified portal layer is shown in the following figure 4 . 


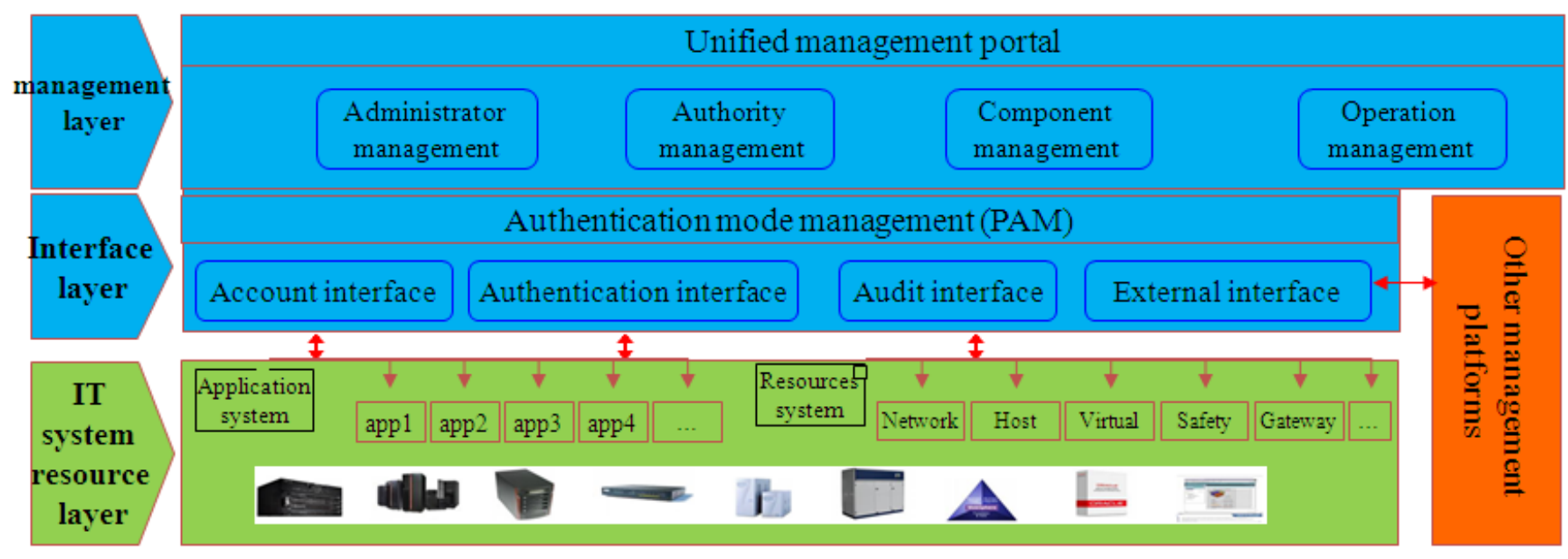

Figure4: Schematic design of the unified portal layer architecture

The management layer is mainly used for the corresponding maintenance management. When user login, application system validate ticket (certification) as the single user authentication credentials, make a unified access entrance to avoid duplicated login of different business systems; Interface layer provides interface communication between various applications, including account interface, authentication interface,audit interface and external interface; IT system resource layer support the integration of various business systems.

2) vNUMA optimization

Through vNUMA (Virtual Non Uniform Memory Access) technology, vNUMA hypervisor(middleware layer) creates a NUMA topology, which can reflect the topology of underlying physical server, and allows the cloud host operating system to access memory and processor intelligently. vNUMA topology of cloud host will simulate the topology structure of host machine, and feedback the topology structure to the applications of cloud host, for the applications to optimization NUMA architecture.

3) Hot Upgrade

Upgrade the virtual machine (do not need to stop the virtual machine) in case of business system online, such as increasing the number of vCPU(Virtual machine CPU) and the capacity of vMemory(Virtual Memory). It can be achieved by calling API structure program libvirt (open source virtualization library) of KVM (Keyboard Video Mouse, simulated operation technology). When users need to hot upgrade, Openstack will call live resize interface of libvirt, to conduct hot upgrade operation to the cloud host.

4) MAC binding

Bind MAC address and IP address of the virtual machine.During the operations of virtual machine migration or IP switching etc., business will not be affected by MAC address binding. With MAC address binding, the security of whole cloud data center will be enhanced greatly, which prevent issues like user modifying IP address of virtual machine,appearing of non-external attack or IP address simulation. When the above happens, since MAC address and IP address are binded, the virtual machine network will be cut off, to enhance network security of whole cloud environment.
5) Software defined network dynamic virtual routing

Through the software defined network dynamic routing technology, routing functions are distributed within multiple virtual machines. During the access time, routing is achieved by all virtual machines together, it prevents routing failures which affect access of the integrated system, so as to enhance the security of entire data center.

6) Online adjustment of physical infrastructure

Once IOT system based on cloud data center is built, due to the use of storage virtualization and dual live technologies, when the storage system of cloud data center physical architecture need adjustment, it can be achieved dynamically with business online.

7) Business system migration

During the cloud-transform of data center, there are two ways to migrate business system for the industry, namely, architecture migration and P2V (Physical to virtual) migration. Architecture migration refers that, in the cloud environment of IOT, pre-deploy exactly the same new environment such as virtual machine, operating system, applications and all kinds of runtime environment deployment, then migrate the data of all business systems to the new environment; P2V migration refers that, package the whole system of current physical host, including applications, data, network parameters, then migrate the packaged system to the virtual machine of IOT, and unpack.

8) Fully autonomous controllable

In order to ensure information security, architecture design of the entire IOT control management platform should be completely autonomous controllable. All the hardware use independent intellectual property rights, innovative and qualified products. Software is developed independently which is very important for the control and management platform of IOT. It can be customized according to the actual features of applications, to meet the personalized business requirements of enterprise.

\section{Summary}

In view of the information island of scattered business systems caused by the construction mode of "project" by current enterprise, building private cloud computing platform 
to integrate all business system servers, storage and network resources, carrying on the transformation to all business systems based on the cloud platform, and then implementing the "IOT applications" is the necessary approach to deal with the problems from various enterprise, such as resources cannot be shared between application systems, data cannot be interacted etc. Along with the landing of scaled cloud services, another innovative technology get the chance to explode, which is the big data. Based on IOT applications with cloud computing, to realize the data exchange and interoperability, promote the benign development of big data applications, equipped enterprises with the ability to establish perfect big data analysis system,there by the competitiveness of enterprise and service of government can be truly enhanced.

\section{References}

[1] Li Tao,Chen Wei-wei,Li Zhi-gang,Liu Tao. "OpenStack Research and comparison of open source platform",Journal of Military Communications Technology,2015(02).

[2] Wang xuedong. "Information resource management for manufacturing oriented enterprises",Manufacture Information Engineering of China,2011(04).

[3] Yan Yan, "The application and future trend of office automation",Guizhou Electric Power Technology,2013(11).

[4] Liang Rui. "Application of Internet of things technology in the supply chain of dangerous goods", Beijing Jiaotong University,2011.

[5] Zhu Ming. "Application of grid computing on-demand IT infrastructure construction",Geomatics World,2005(01).

[6] Wang Ya Mei,Ren Hao Li. "Research on the design of integrated service processing office automation system", Education Teaching Forum,2012(25).

[7] Zhou Y, Chen G, Liu G. "Intelligent Sensor System UPnp Networks Interface and Device Selection Realization".Advanced Materials Research.2013(718720).

[8] Nieves AR. "A UPnp Service to Control and Manage IEEE 1451 Transducers in Control Network", Transactions on Instrumentation and Measurement, 2012,61(3):791-800.

[9] Si Yifang, Sun Manli, Wang Nan. "Realizing Intelligence and Energy Saving of Residence Based on Wireless Sensor Network", 2010,46-48.

[10] Long Fan. "The Research and Realization of Intellgent Home Residential Gateway Based on ZigBee Techniques",Beijing2010.

[11] Evan W, Leilani B, Garret C, eta. "Building the Internet of things using RFID:The RFID ecosystem experience", IEEE Internet Computing, 2009,13(3):48-50.

[12] Amardeo C,Sarma J G. "Identities in the Future Internet of Things", wireless Pers Community,2009(49). 